East African Medical Journal Vol. 85 No. 6 June 2008

BACTERIOLOGICAL AND CLINICAL EVALUATION OF TWELVE CASES OF POST-SURGICAL SEPSIS OF ODONTOGENIC TUMOURS AT A REFERRAL CENTRE

L.O. Egwari, PhD, N.N. Nwokoye, MSc., AMILS, Department of Botany and Microbiology, Faculty of Science, University of Lagos, Akoka, Lagos, Nigeria, B. Obisesan, DDPHRCS (Engl), FPMCN, Department of Oral and Maxillofacial Surgery, A.O. Coker, PhD, FRCPath (UK), FAS (Nigeria), F.O. Nwaokorie, MSc. FIMLS, Department of Medical Microbiology and Parasitology and K.O. Savage, MSc, FMCDS, EWACS, Department of Preventive Dentistry, College of Medicine, University of Lagos, Lagos, Nigeria

Request for reprints to: Dr. L.O. Egwari, Department of Botany and Microbiology, Faculty of Science, University of Lagos, Akoka, Lagos, Nigeria

\title{
BACTERIOLOGICAL AND CLINICAL EVALUATION OF TWELVE CASES OF POST-SURGICAL SEPSIS OF ODONTOGENIC TUMOURS AT A REFERRAL CENTRE
}

\section{L.O. EGWARI, N.N. NWOKOYE, B. OBISESAN, A.O. COKER, F.O. NWAOKORIE and K.O. SAVAGE}

\begin{abstract}
Objective: To determine the bacterial aetiology of sepsis occurring following surgery of odontogenic tumours and assess the effect of prompt and proper antibiotic usage.

Design: A prospective study.

Setting: A tertiary referral centre in Lagos, Nigeria.

Subjects: Twelve patients with odontogenic tumours that developed sepsis postoperatively. Eight of the patients were referred from private hospitals, the remaining were in-patients who sought for alternative medical attention following interruption of health care services at the referral centre. Intervention: Adequate review of patient's medical history, bacteriological investigations and antibiotic therapy.

Main outcome measures: Bacteriological and clinical cure following antibiotic therapy based on susceptibility test results.

Results: Two categories of patients were identified; those who completed the course of antibiotics prescribed post-surgery and patients who failed to conform to antibiotic prescription. Sepsis developed in the non-compliance group much earlier than in the group that complied $(p<0.001)$. Infections were polybacterial with aerobes accounting for $77.4 \%$ ( $\alpha$-haemolytic streptococci $29.0 \%$, Streptococcus pyogenes $16.1 \%$, Staphylococcus aureus $16.1 \%$, diphtheroids $9.7 \%$, Klebsiella pneumoniae $6.5 \%$ ) and anaerobes $22.6 \%$ (Porphyromonas gingivalis $9.7 \%$, Peptostreptococcus spp. $6.5 \%$, Prevotella melaninogenica $3.2 \%$, Clostridium perfringens $3.2 \%$ ). Mixed aerobic and anaerobic aetiology occurred more in osteosarcoma and fibrosarcoma. Clostridium perfringens was isolated from a case of osteosarcoma with necrotic tissues. The anaerobic bacteria were $100 \%$ sensitive to metronidazole, ciprofloxacin and augmentin, $65-85 \%$ sensitivity to ampicloxacillin, amoxicillin and erythromycin. Over $92 \%$ of the streptococci were sensitive to the $\beta$-Lactams contrast low susceptibility with S. aureus and K. pneumoniae.

Conclusion: Interruption of healthcare service was the sole factor identified in the development of sepsis as the patients could not be monitored to ensure compliance to prescription.
\end{abstract}

\section{INTRODUCTION}

A review of the database suggests increased occurrence of tumours in the head and neck region, most, of which are odontogenic in origin $(1,2)$. While there is a uniform consensus to surgery as the cornerstone for the treatment of tumours and carcinomas $(1,3)$ with radiotherapy indicated 
post-operatively (3) opinion differs with respect to the use of antibiotherapy in local odontogenic diseases (4). Many practitioners have recommended adequate follow-up for tumour patients for early detection of complications and recurrences. This has been shown to increase treatment success rate $(5,6)$. One of the complications reported is secondary microbial infection. For instance, a case of adenocystic carcinoma in the left bronchus was reported to have been complicated with tracheo bronchial aspergillosis (6), while Zwahlen and Gratz presented a 13 year follow-up case of a 44 year old male patient with maxillary ameloblastoma who died of multiple metastases even after repeated surgical intervention and radiotherapy. The patient's condition was further compromised by chronic exudative otitis media (4).

The oral surface and its tissues contain approximately $10^{11}$ to $10^{12}$ microorganisms / g of tissue of which $90 \%$ are anaerobes (7). Thus the risk for postoperative infection with endogenous microorganisms is high (1-5\% in clean operative procedures and $27 \%$ in dirty procedures) (8). This informs our routine practice of giving prophylactic postoperative antibiotic cover to our patients. However, where sepsis developed after initial antibiotic coverage, change in therapy was usually governed by laboratory results on the susceptibility of the pathogen(s). In this prospective study, we examined circumstances that led to postsurgical sepsis in twelve patients with odontogenic tumours, determined the bacteriological features of the sepsis and assessed the effect of prompt and proper antibiotic usage.

\section{MATERIALS AND METHODS}

Between February 2002 and June 2004 twelve patients that developed post-surgical sepsis from odontogenic tumours were examined and treated at our centre. Eight of the patients were referred cases from private hospitals; the cases included osteosarcoma (five patients), fibrosarcoma (two patients), and a patient with ameloblastoma. Follow-up records were not available as the patients only revisited on development of complications. The patient's case history were reviewed and found to be of low economic status and in most cases did not complete their medication. Sepsis developed in these patients three to six weeks post operation with an average duration of 3.8 weeks.

The remaining patients (two cases each of adenocystic carcinoma and maxillary \{periodontal\} cysts) were first seen and treated at the Lagos University Teaching Hospital, Lagos and received resection alone or with radiotherapy and postoperative antibiotic coverage. Antibiotics given empirically were a combination of ampicloxacillin (500mg 6 hourly for 1 week), or amoxicillin (500mg 8 hourly for 1 week) and metronidazole (400mg 8 hourly for 1 week). The patients were discharged untimely from hospital owing to industrial dispute in the hospital that resulted in temporary suspension of health care services. It was therefore difficult to monitor patient's compliance to prescriptions though three of the patients claimed to have completed their medication. The patients reported back with septic complications one to two months following surgery. The time lapse before sepsis developed in patients that completed their antibiotic course and those that did not was analysed for statistical significance using the students t-test at $\mathrm{p}=0.001$.

The patients were re-examined and detailed bacteriological investigations carried out. The patients were subsequently placed on antibiotic therapy based on the results of susceptibility for each pathogen.

Specimens collected for bacteriological investigations included needle aspiration of pus, swab specimens and curettage. Alginate swab specimens were transported in Portagerm tube (Biomerieux) and Amies transport medium (Oxoid). The curettage samples were transported in anaerobic vacutainer vials, while needle aspirates were transported in Portagerm vials. All specimens were processed within 1-2 hours of collection. For aerobic culture the following media were used; Sheep blood agar plates, MacConkey agar (Lab $\mathrm{M})$, and Brain heart infusion agar (Oxoid). Anaerobic cultures were set up in non-selective anaerobic blood agar plates supplemented with vitamin $\mathrm{K}_{1}(\mathrm{l} \mu \mathrm{g} / \mathrm{ml})$, and L-cysteine hydrochloride $(2 \mu \mathrm{g} / \mathrm{ml})$, neomycin blood agar $(100 \mu \mathrm{g} / \mathrm{ml}$ neomycin), kanamycin blood agar $(75 \mu \mathrm{g} / \mathrm{ml}$ kanamycin) and reinforced clostridial medium (Oxoid). For both primary isolation and preliminary identification of anaerobes, metronidazole discs $(5 \mu \mathrm{g})$ and gentamicin discs $(10 \mu \mathrm{g})$ were placed on the surface of the media for anaerobic incubation. Anaerobic incubation was achieved at $10 \% \mathrm{CO}_{2}$ and $10 \% \mathrm{H}_{2}$ using the GasPak anaerobic jar system (Oxoid). Inoculated plates were incubated at $37^{\circ} \mathrm{C}$ for between 18-72 hours for aerobic cultures and 4-10 days for anaerobic cultures. Cultures were purified and identified by conventional methods for both aerobic and anaerobic bacteria $(9,10)$. 
The isolated pathogens were subjected to antibiotic susceptibility testing using methods described by the National Committee for Clinical Laboratory Standards (11). Antibiotics tested were ampicloxacillin $(10 \mu \mathrm{g})$, amoxicillin $(25 \mu \mathrm{g})$, augmentin (amoxicillin $20 \mu \mathrm{g}$ and clavulanic acid $(10 \mu \mathrm{g})$, erythromycin $(15 \mu \mathrm{g})$ and ciprofloxacin $(10 \mu \mathrm{g})$ for both aerobic and anaerobic isolates. Ceftriaxone $(30 \mu \mathrm{g})$ activity was evaluated only against the aerobic bacteria while penicillin (4 IU) and metronidazole $(5 \mu \mathrm{g})$ activities were determined only against the anaerobic bacteria. The results of the susceptibility tests were controlled with S. aureus NCTC 6751 for the aerobic bacteria and Prevotella bivia ATCC 5540 for the anaerobic bacteria. Beta-lactamase production in isolates was determined using the nitrocefin disks (Cefinase disks, BBL Microbiology Systems, Cockeysville, MD) (12) and the starch paper technique that detects penicillinase activity qualitatively (13).

\section{RESULTS}

Infections were polybacterial with aerobes accounting for $77.4 \%$ ( $\alpha$-haemolytic streptococci $29.0 \%$, Strep. pyogenes $16.1 \%$, S. aureus $16.1 \%$, diphtheroids $9.7 \%$, K. pneumoniae 6.5\%) and anaerobes $22.6 \%$ (Porp. gingivalis 9.7\%, peptosreptococcus spp. 6.5\%, P. melaninogenica $3.2 \%$, C. perfringens $3.2 \%$ ). Mixed aerobic and anaerobic infections occurred more in osteosarcoma and fibrosarcoma, however, the mean number of isolates per clinical entity was fibrosarcoma; four, ameloblastoma; three, maxillary cyst; three, osteosarcoma; three and adenocystic carcinoma; one. Monoculture of $C$. perfringens was obtained in a case of osteosarcoma while maxillary cyst cultures were solely of aerobic bacteria (Table 1).

The antibiotic susceptibility patterns of the oral pathogens are given in Table 2 . All the isolates were sensitive to ciprofloxacin while augmentin and metronidazole produced $100 \%$ activity against the anaerobes. Porphyromonas gingivalis and C.perfringens were sensitive to all the antibiotics including ampicloxacillin and amoxicillin. Staphylococcus aureus, K. pneumoniae and P. melaninogenica were resistant to the penicillins, with some strains of $S$. aureus and K. pneumoniae resistant to ceftriaxone. Beta-lactamase production was demonstrated in the aerobic bacteria

Table 3 reviewed the clinical data of the 12 patients with odontogenic tumours treated for post-surgical sepsis. Eight of the patients who did not comply with prescription (non-compliance group) developed sepsis 3-5 weeks (Mean, 3.5 weeks; SD, 0.71) while the remaining four patients who completed their antibiotic course (the compliant group) developed sepsis 5-8 weeks (Mean, 6.25 weeks; SD, 1.09).

Table 1

Bacterial type and distribution in post-surgical sepsis of odontogenic tumours

\begin{tabular}{|c|c|c|c|c|c|c|c|}
\hline \multirow[t]{2}{*}{ Isolates } & \multirow[t]{2}{*}{ Fibro. } & \multirow[t]{2}{*}{ Amel. } & \multirow[t]{2}{*}{ M. cyst } & \multirow[t]{2}{*}{ Adeno. } & \multirow[t]{2}{*}{ Osteo. } & \multicolumn{2}{|c|}{ Isolation } \\
\hline & & & & & & No. & $(\%)$ \\
\hline \multicolumn{8}{|l|}{ Anaerobes } \\
\hline C. perfringens & 0 & 0 & 0 & 0 & 1 & 1 & 3.2 \\
\hline Porp. gingivalis & 1 & 0 & 0 & 1 & 1 & 3 & 9.7 \\
\hline P. melaninogenica & 0 & 1 & 0 & 0 & 0 & 1 & 3.2 \\
\hline Peptostreptococci & 1 & 0 & 0 & 0 & 1 & 2 & 6.5 \\
\hline \multicolumn{8}{|l|}{ Aerobes } \\
\hline \multicolumn{8}{|l|}{$\alpha$-haemolytic } \\
\hline Streptococci & 2 & 0 & 1 & 1 & 5 & 9 & 29.0 \\
\hline Strep. pyogenes & 1 & 1 & 0 & 0 & 3 & 5 & 16.1 \\
\hline S. aureus & 0 & 0 & 1 & 0 & 4 & 5 & 16.1 \\
\hline K. pneumoniae & 1 & 1 & 0 & 0 & 0 & 2 & 6.5 \\
\hline Diphtheroids & 2 & 0 & 1 & 0 & 0 & 3 & 9.7 \\
\hline
\end{tabular}

Fibro = Fibrosarcoma; Amel = Ameloblastoma; M.cyst = Maxillary cyst; Adeno = Adenocystic carcinoma; Osteo $=$ Osteosarcoma. 
Table 2

Antibiotic susceptibility pattern of the isolated pathogens

\begin{tabular}{|c|c|c|c|c|c|c|c|c|}
\hline \multirow[t]{2}{*}{ Isolates (No. tested) } & \multicolumn{8}{|c|}{ Number sensitive to antibiotics } \\
\hline & Apc. & Amx. & Pen. & Amc. & Ery. & Cip. & Cfx. & Met. \\
\hline \multicolumn{9}{|l|}{$\alpha$-haemolytic } \\
\hline Streptococci (9) & 8 & 8 & - & 9 & 8 & 9 & 9 & - \\
\hline Strep. pyo genes (5) & 5 & 5 & - & 5 & 5 & 5 & 5 & - \\
\hline S. aureus (5) & 0 & 0 & - & 2 & 2 & 5 & 2 & - \\
\hline K. pneumoniae(2) & 0 & 0 & - & 1 & - & 2 & 1 & - \\
\hline Porp. gingivalis (2) & 2 & 2 & 2 & 2 & 2 & 2 & - & 2 \\
\hline P. melaninogenica $(1)$ & 0 & 0 & 0 & 1 & 1 & 1 & - & 1 \\
\hline Peptostreptococci (2) & 1 & 1 & 0 & 2 & 1 & 2 & - & 2 \\
\hline C. perfringens (1) & 1 & 1 & 1 & 1 & 1 & 1 & - & 1 \\
\hline
\end{tabular}

Apc. $=$ Ampicloxacillin; Amx. $=$ Amoxicillin; Pen. $=$ Penicillin; Amc. $=$ Augmentin; Ery. $=$ Erythromycin; Cip. = Ciprofloxacin; Cfx. = Ceftriaxone; - = not tested.

Table 3

Review of clinical data of patients with odontogenic tumours treated for post-surgical sepsis

\begin{tabular}{|c|c|c|c|c|c|c|c|}
\hline \multicolumn{2}{|c|}{ Clinical condition } & \multicolumn{3}{|c|}{ Case review } & \multirow{2}{*}{$\begin{array}{c}\text { Appr. DSD } \\
\text { (weeks) }\end{array}$} & \multicolumn{2}{|c|}{ Treatment given post-sepsis } \\
\hline & & Hosp & POC & PCP & & DRN & Antibiotics given \\
\hline \multirow[t]{7}{*}{ Osteosarcoma } & 1 & $\mathrm{PH}$ & Apc 500mg 6h/7D & No & 5 & + & Cip 500mg bid/7D \\
\hline & & & Met $400 \mathrm{mg} 8 \mathrm{~h} / 7 \mathrm{D}$ & & & & Met 400mg 8h/7D \\
\hline & 2 & $\mathrm{PH}$ & ditto & No & 3 & + & ditto \\
\hline & 3 & $\mathrm{PH}$ & ditto & No & 3 & + & ditto \\
\hline & 4 & $\mathrm{PH}$ & ditto & No & 4 & + & ditto \\
\hline & 5 & $\mathrm{PH}$ & Apc 500mg 6h/7D & Yes & 6 & + & Apc 500mg 6h/7D \\
\hline & & & Met 400mg 8h/7D & & & & Met 400mg 8h/7D \\
\hline \multirow[t]{3}{*}{ Fibrosarcoma } & 1 & PH & ditto & No & 3 & + & Cip 500mg bid/7D \\
\hline & 2 & $\mathrm{PH}$ & Amx 500mg 8h/7D & No & 3 & + & Cip 500mg bid/7D \\
\hline & & & Met 400 8h/7D & & & & Met 400mg 8h/7D \\
\hline \multirow{2}{*}{\multicolumn{2}{|c|}{ Ameloblastoma }} & PH & Amx 500mg 8h/7D & No & 3 & + & Amc $625 \mathrm{mg}$ bid/7D \\
\hline & & & Met 400mg 8h/7D & & & & \\
\hline \multirow[t]{2}{*}{ Ad. Carcinoma } & 1 & LUTH & Apc 500mg 6h/7D & Yes & 8 & + & Met 400mg 8h /7D \\
\hline & 2 & LUTH & ditto & Yes & $S$ & + & Apc 500mg 6h/7D \\
\hline \multirow[t]{2}{*}{ Maxillary cyst } & 1 & LUTH & ditto & No & 4 & + & Cip 500mg bid/7D \\
\hline & 2 & LUTH & ditto & Yes & 6 & + & Apc 500mg 6h/7D \\
\hline
\end{tabular}

$\mathrm{P}<0.001$ ( $\mathrm{t}$-test) comparing differences in duration for development of sepsis in patients who received and complied with antibiotic therapy and patients that failed to comply with antibiotic prescription. Hosp $=$ Hospital, POC $=$ Post-operative antibiotic coverage, PCP = Patients compliance to prescription, DSD = Duration sepsis developed, DRN = Drainage, ditto = as indicated above. PH; Private hospital, LUTH = Lagos University Teaching Hospital, Apc = Ampicloxacillin, Amx = Amoxicillin, Met = Metronidazole Amc $=$ Augmentin, Cip $=$ Ciprofloxacin,$+=$ drainage was carried out, No $=$ No compliance to antibiotic prescription, Yes $=$ Compliance to antibiotic prescription, $6 \mathrm{~h} / 7 \mathrm{D}=$ given 6 hourly for 7 days, $8 \mathrm{~h} / 7 \mathrm{D}=$ given 8 hourly for 7 days, bid = given twice daily. 
Analysis of the data showed that non-compliance to prescription proceeded to early development of sepsis $(p<0.001)$. The choice of antibiotics was based on the bacteriology of the sepsis and the determined antibiotic susceptibility patterns of the infecting flora. Ciprofloxacin was given in addition with metronidazole when $S$. aureus or K. pneumoniae was one of the infecting flora. Single drug therapy was given in case of mono-cultures or when all infecting pathogens were sensitive to a particular antibiotic. Clinical response in all patients was good and no relapses occurred after two months of follow-up.

\section{DISCUSSION}

Malignancies are immunosuppressive conditions and usually predispose to infection with both pathogenic and opportunistic microorganisms. Odontogenic tumours as well as their treatment modalities compromise the patient's immunity and increase the risk of infection with both endogenous and exogenous flora. The isolation of aerobic and anaerobic bacteria from the septic cases studied emphasizes the importance of microorganisms in the healing process of these tumours. Other studies have also described microbial association with odontogenic tumours $(4,6)$. In the two cited studies, adenocystic carcinoma predisposed a patient to pulmonary aspergillosis (6) while maxillary ameloblastoma in a 44 year old patient was complicated with chronic exudative otitis media (4). These findings are sufficient evidence to include antibiotics as part of the regimen for managing odontogenic tumours and related malignancies. Furthermore, early sepsis developed in patients who did not comply with antibiotic prescription. It may be erroneous to conclude that treatment failure due to the presence of antibiotic resistant strains was responsible for sepsis in the group that completed their antibiotic course. If this was the case sepsis could have developed much earlier. Other factors such as good oral hygiene and regular mouth rinses with saline are equally essential for prompt healing of surgical odontogenic wounds. Where these are ignored, the wound can be re-infected either with endogenous or exogenous bacteria four weeks or more after completion of initial antibiotic therapy. The effectiveness of the initial antibiotic therapy given can be substantiated on the ground of the similarity in antibiotics given pre- and post sepsis. When patient's therapy was supervised sepsis resolved and healing process was facilitated.

The high susceptibility of the streptococci to the penicillins justified the continued use of these agents in the treatment of oro-facial diseases of microbial aetiology. The activity of the penicillins may be limited by $\beta$-lactamase production by indigenous oral anaerobes especially Prevotella and Porphyromonas that subsequently provide protection for non $\beta$-lactamase producing Streptococcus spp. $(12,14)$. It was therefore necessary to combine metronidazole and ampicloxacillin following drainage of suppurative tissues. This conforms to our usual clinical practice to give low cost drugs where laboratory investigations support their activities. For purely anaerobic aetiology metronidazole was given. Ciprofloxacin was preferred over augmentin and was given in combination with metronidazole where the aetiology is polybacterial and include a $\beta$-lactamase producing bacterium such as $S$. aureus or K. pneumoniae. From our experience, this combination has yielded greater success rate, but this has not necessitated abandoning the routine practice of giving ampicloxacillin-metronidazole combination post-surgery. The fluoroquinolones are relatively more expensive drugs than most oral penicillins and the possibility of patients not completing the full dosage on cost ground exist. The remote consequence may be emergence of ciprofloxacin resistant bacterial strains which we try to safeguard. Not withstanding, the excellent in vitro activity of ciprofloxacin against both aerobic and anaerobic bacteria isolated in this study may make the fluoroquinolones ready alternatives to mainstays in our centre.

Beta-lactamase production in S. aureus and K. pneumoniae in this study accounts for their high level resistance to $\beta$-lactam antibiotics $(15,16)$. However, their high sensitivity to ciprofloxacin was associated with therapeutic success achieved when ciprofloxacin was given to patients with S. aureus and K. pneumoniae infections. The only Clostridium perfringens isolate was very sensitive to the penicillins, ciprofloxacin and metronidazole. Thus, early initiation of antibiotic therapy could have prevented C. perfringens myonecrosis as presented in one of the patients with osteosarcoma. This patient on readmission was treated with a combination of ampicloxacillin 500mg six hourly and metronidazole 
$400 \mathrm{mg} 8$ hourly for seven days. Though the isolate was sensitive to ampicloxacillin, metronidazole was included in the regimen to suppress the population of indigenous anaerobic bacteria with the propensity to produce large amount of $\beta$-lactamases to which ampicloxacillin is susceptible. Clostridium perfringens has been described together with $S$. aureus as a leading pathogen in necrotising soft tissue infections $(17,18)$.

The diversity of microbial aetiology in sepsis complicated surgical procedures of the odontogenic tumours justified the need to give antibiotics post-operatively. The success of this practice will however be dependent on patients' compliance to prescription. Absence of adequate follow-up as observed in this study was responsible for noncompliance, and consequently the development of septic complication which is unusual with in-patients at our centre where compliance to antibiotics prescription is ensured by nurses.

\section{ACKNOWLEDGEMENTS}

To the Academic Planning Unit of the University of Lagos, Lagos, Nigeria for the support grant for this study. We also acknowledge the technical assistance of Mrs. V.O. Bakare and Miss A. Izobo of the Department of Botany and Microbiology, Faculty of Science, University of Lagos, Lagos, Nigeria.

\section{REFERENCES}

1. Adebayo, E.T., Ajike, S.O. and Adekeye, E.O. Odontogenic tumours in children and adolescents: a study of 78 Nigerian cases. J. Cranio-Maxillofac. Surg. 2002; 30: 267-272.

2. Avon, S.L., McComb, J. and Clokie, C. Ameloblastic carcinoma: Case report and literature review. J. Can. Dent. Assoc. 2003; 69: 573-576.

3. Maciejewski, A., Szymczyk, C. and Wiergon, J. Outcome of surgery for adenoid cystic carcinoma of head and neck region. J. Cranio-Maxillofac. Surg. 2002; 30: 59-61.

4. Zwahlen, R.A. and Gratz, K.W. Maxillary ameloblastomas: A review of the literature and of a 15-year database. J. Cranio-Maxillofac. Surg. 2002; 30: 273-279.

5. Witten, J., Hybert, F. and Hansen H.S. Treatment of malignant tumours in the parotid glands. Cancer, 1990; 65: 2515-2520.
6. Navarro, F., Vazquez, J., Cicero, R. and Legaspi, A. Adenocystic bronchial carcinoma associated with tracheobronchial aspergillosis. Ann. Med Assoc. Med Hosp. ABC, 1997; 42: 27-29.

7. Bartlett, J.G. and Gorbach, S.L. Anaerobic infections of the head and neck. Otolaryngol. Clin. North Amer. 1976; 9: 655-678.

8. Nichols, R.L. Postoperative infections in the age of drug-resistant gram-positive bacteria. Amer. J. Med. 1998; 104: 11S-16S.

9. Cowan, S.T. Cowan and Steel Manual for the Identification of Medical Bacteria. $2^{\text {nd }}$ edn., Cambridge: Cambridge University Press, 1974.

10. Holdeman, L.V., Cato, E.P. and Moore, W.E.C. Anaerobe Laboratory Manual, Virginia Polytechnic Institute and State University. Blacksburg, Virginia, 1979; 134-137.

11. National Committee for Clinical Laboratory Standards: Performance standards for antimicrobial susceptibility testing. Second information supplement M10-S2, NCCLS, Villanova, Pa 1987.

12. Fosse, T., Madinier, I., Hitzig, C. and Charbit, Y. Prevalence of $\beta$-lactamase-producing strains among 149 anaerobic gram-negative rods isolated from periodontal pockets. Oral Microbiol. Immunol. 1999; 14: 352-357.

13. Odugbemi, T.O., Hafiz, S. and McEntegart, M.G. Penicillinase producing Neisseria gonorrhoeae: Detection by starch paper technique. Brit. Med. J. 1977; 2: 550-552.

14. Riesbeck, K. Paronychia due to Prevotella bivia that resulted in amputation: Fast and correct bacteriological diagnosis is crucial. J. Clin. Microbiol., 2003; 41: 4901-4903.

15. Egwari, L.O. and Nwachukwu, A.E. Distribution and susceptibility of methicillin resistant staphylococci to the quinolones and four other antibiotics. J. Med. Lab. Sci. 1994; 4: 81-86.

16. Meyer, K.S., Urban, C., Eagan, J.A., et al. Nosocomial outbreak of Klebsiella infection resistant to lategeneration cephalosporins. Ann. Intern. Med. 1993; 119: 353-358.

17. Kingston, D. and Seal, D.V. Current hypotheses on synergistic microbial gangrene. Brit. J. Surg. 1990; 77: 260-264.

18. Regev, A., Weinberger, M., Fishman, M., et al. Necrotizing fasciitis caused by Staphylococcus aureus. Eur. J. Clin. Microbiol. Infect. Dis. 1998; 17: 101-103. 